\title{
NOBLES Y MONJES: LOS OSORIO VILLAFRANQUINOS Y LOS MONASTERIOS BERCIANOS (SIGLOS XIV-XV)
}

\author{
POR \\ Gregoria CAVERo DomíngUeZ \\ Universidad de León \\ g.cavero@unileon.es
}

\section{RESUMEN}

A lo largo de los siglos bajomedievales se produjo en el noroeste leonés una especial alianza entre la nobleza, representada por el linaje Osorio, de la casa condal de Lemos, y el monacato tradicional y mendicante. Es nuestro objetivo analizar el papel de la poderosa nobleza que culminaría su expansión económica y lograría encontrar un lugar para el eterno descanso de sus miembros dentro de los numerosos centros monásticos bercianos, masculinos y femeninos, urbanos y rurales: Santa María de Carracedo y San Francisco de Villafranca, entre otros.

PALABRAS CLAVE: Nobleza; Osorio; encomienda; monasterios; El Bierzo; capillas funerarias.

\section{NOBLES AND MONKS: THE OSORIOS FROM VILLAFRANCA AND THE MONASTERIES IN EL BIERZO (14TH-15TH CENTURIES)}

\begin{abstract}
Throughout the Middle Ages, in the northwest of the present day province of León, there occurred a special alliance between the nobility, represented by the Osorios, from the Count House of Lemos, and the traditional monasticism and mendicant friars. The powerful nobility would reach the peak of their economic growth and find a place for the eternal rest of their members in the numerous Bierzo monastic centres, whether male or female, urban or rural, like Santa María de Carracedo and San Francisco de Villafranca, among others.
\end{abstract}

KEY WORDS: Nobility; Osorio; encomienda; monasteries; El Bierzo; funereal chapels.

$\begin{array}{ll}\text { Recibido/Received } & \text { 09-11-2014 } \\ \text { Aceptado/Accepted } & \text { 24-07-2015 }\end{array}$

Frente a la crisis del monacato tradicional, especialmente de base benedictina, la época bajomedieval presenta el auge del modelo mendicante. El protagonismo de los monasterios en El Bierzo medieval, sus abundantes fondos documentales -algunos, originales; otros, procedentes de tumbos- constituyen la base del análisis de monjes y nobles en la época bajomedieval en el citado espacio comarcal leonés que abordaremos. Para ello nos serviremos de fuentes documentales tanto publicadas como inéditas. ${ }^{1}$

1 Nos referimos especialmente a: Cavero Domínguez, G. y Domínguez Sánchez, S. 2000. Colección Documental de la Catedral de Astorga (1300-1499), vol. III, Astorga; Cavero Domínguez, G. 1994. Catálogo del Monasterio de San Miguel de las Dueñas, que incluye: "Monasterio de San Guillermo de Villabuena (1172-1527)", pp. 15-53, León; Martínez Martínez, M. 1997-1999. Cartulario de Santa María de Carracedo (992-1500) (=I C.), 2 vols., Ponferrada: Instituto de Estudios
Los nobles, en este caso, pertenecían al linaje Osorio. ${ }^{2}$ Los

Bercianos; Quintana Prieto, A. 1971. Tumbo viejo de San Pedro de Montes, León; Jiménez Suárez, M. J. 2005. Colección documental de San Andrés de Espinareda (1043-1428), León.

Hay también historias monásticas: González González, F. (ed.) 1993. Memorial de la fundación y dotación del antiguo y devoto monasterio de Santa María de Carracedo, Zaragoza. En el siglo XIX, el cisterciense gallego Ambrosio Delgado se convirtió, tras la exclaustración, en capellán de las monjas de Almázcara y escribió la Historia de San Miguel de las Dueñas, que se halla, de forma manuscrita, en el archivo monástico.

Se encuentra sin catalogar el fondo documental del convento de la Concepción de Villafranca, con documentación desde el siglo XV. Igualmente recurrimos al Archivo Ducal de la Casa de Alba (Madrid), especialmente el fondo de la Casa de Lemos. Por su importancia es también necesario mencionar el Archivo Ducal de la Casa de Medina Sidonia (Sanlúcar de Barrameda).

2 Sobre el linaje, remitimos al siguiente trabajo: Martín Fuertes, J. A. 1988. De la nobleza leonesa. Los Osorio y el Marquesado de Astorga, 
monjes eran de procedencia más plural: del monacato tradicional de base benedictina, como San Pedro de Montes y San Andrés de Espinareda; de la reforma cluniacense, como Santa María de Cluny de Villafranca; y de la reformada línea cisterciense, como Carracedo, San Miguel de las Dueñas y San Guillermo de Villabuena. En el siglo XIII los mendicantes se asentaron en El Bierzo, especialmente en Villafranca y, posteriormente, en Cabeza de Alba. Ya a comienzos del siglo XVI, bajo la tutela directa del marquesado de Villafranca, surgió el convento concepcionista de la villa.

La relación de monjes y monjas con los Osorio constituye el análisis que presentamos, estructurado en cuatro apartados: en primer lugar, el enriquecimiento de los Osorio a costa de los patrimonios monásticos; en segundo, la búsqueda de un descanso para la eternidad y la demanda de auxilios espirituales; en tercer lugar, el apellido Osorio entre los monjes bercianos; $y$, finalmente, las fundaciones conventuales en que de forma directa participaron los miembros de dicho linaje.

\section{USURPACIÓN DE PROPIEDADES. LA ENCOMIENDA Y LOS ABADES COMENDATARIOS}

La crisis del monacato berciano tradicional se dejó sentir básicamente durante los siglos bajomedievales, especialmente desde los críticos años centrales del siglo xIV. La solución por la que optaron los monasterios fue la encomienda: ${ }^{3}$ la entrega de propiedades a nobles y caballeros con el fin de obtener una defensa de sus patrimonios y vasallos. La solución comportaba la figura de los encomenderos, caracterizados por usos y abusos, ante los que los monjes se vieron obligados a recurrir a la monarquía y, en ocasiones, a Roma. Las cartas de amparo solicitadas trataron de frenar los abusos y obligaron a los monasterios a intentar la recuperación patrimonial y utilizar otro sistema menos conflictivo y más provechoso. Por ello desde finales del siglo xIV se generalizó especialmente el sistema de contrato de foro, cuyas rentas anuales llegaban más fluidamente al monasterio.

La crisis que generaron -o a la que, al menos, contribuyeron- las encomiendas, los encomenderos y los abades comendatarios tuvo también su repercusión en Roma, que trató de remediar lo que ya parecía insostenible. En 1484 unas litterae solemnes de Sixto IV confirmaban a la catedral de Astorga y a tres de sus más señeros monasterios diocesanos (San Pedro de Montes, San Martín de Castañeda y San Andrés de Espinareda) sus posesiones, y prohibían expresamente que ningún magnate tuviese en encomienda dichos bienes, puesto que, con tal pretexto, se adueñaban de ellos. ${ }^{4}$

León. Para el marquesado villafranquino, Franco Silva, A. 1981 "Bienes, rentas y vasallos del Señorío de Villafranca del Bierzo", Archivos Leoneses, 69: 39-72; y Franco Silva, A.1982 "El Señorío de Villafranca del Bierzo", Boletín de la Real Academia de la Historia, CLXXIX: 35-160. Igualmente debe contextualizarse con el ámbito gallego, especialmente a través de los trabajos de Pardo de Guevara y Valdés, E., 2012. Los linajes, parentelas y grupos de poder. Aportaciones a la historia social de la nobleza bajomedieval gallega, Madrid. Y, del mismo autor, la pequeña biografía de 2008, Don Pedro Álvarez Osorio. Un noble berciano del siglo XV, Ponferrada.

3 Para su comprensión remitimos al estudio de Santos Díez, J., 1961. La Encomienda de monasterios en la Corona de Castilla, siglos $X-X V$, Roma.

4 Cf. Cavero Domínguez, G. y Domínguez Sánchez, S. 2000, doc. 2.223, pp. 578-582.

\subsection{Villabuena}

En 1229 Alfonso IX dotaba el monasterio que su exesposa Teresa de Portugal fundaba en Villabuena. La carta dotacional original del monarca leonés está en el fondo documental de la ducal Casa de Alba, en el Palacio de Liria, y allí debió de estar también en la Baja Edad Media y en época moderna ya que fray Tomás de Peralta, autor del tumbo de San Miguel de las Dueñas, no recoge ni la existencia ni el contenido del diploma. ${ }^{5}$

Alfonso IX dotaba al cenobio cisterciense con el cillero de Villabuena y propiedades y vasallos en los lugares de Valtuille de Arriba y Valtuille de Abajo, Corullón, Horta, Otero, Villadecanes, Arborbuena, Quilós, Santa Olaya, Canedo de Arriba y Canedo de Abajo, La Válgoma, Cubillos y Vilela. En 1238 se hizo una relación de sus vasallos, foros, martiniegas y solares, en el ámbito berciano; esta relación se halla en el Archivo de la Casa Ducal de Medina Sidonia. ${ }^{6}$

Un siglo después de su fundación, el papa Benedicto XII dio a Carracedo una conservatoria sobre Villabuena:

"refiere que las abadesas de San Guillermo de Villabuena han hecho foros y otros contratos, etc., en perjuicio de su monasterio y le encarga que con censuras compela a la restitucion de lo asi enagenado". ${ }^{7}$

Ello ponía en evidencia la caótica situación económica del cenobio femenino, que parecía, además, estar muy endeudado. En 1370, la abadesa villabonense, Milia García, hace donación a Alfonso, abad benedictino de San Andrés de Espinareda, de 1.900 mrs. para saldar la deuda contraída con el monasterio espinarense. ${ }^{8}$

En 1431 la abadesa y el convento de San Guillermo de Villabuena, manifestando penuria económica, con autorización del abad de Carracedo y de su visitador, el abad del monasterio de Nogales, entregaban a Pedro Álvarez Osorio, señor de Cabrera y Ribera, varios de los lugares dotacionales registrados en el documento alfonsino. En el documento se hace especial referencia a los dos Valtuilles y La Válgoma, que, según la abadesa, estaban perdidos; lo que se traducía en que no percibían rentas desde hacía varios años. Se esperaba del Osorio que los recuperase y, por otra parte, éste pagaría anualmente al monasterio $500 \mathrm{mrs}$. de renta. ${ }^{9}$

Una centuria más tarde el monasterio había desaparecido, invadido por las aguas del Cúa, y sus monjas hacían vida claustral en el monasterio de San Miguel de las Dueñas. El núcleo hacendístico del patrimonio villabonense quedaba en manos de los Osorio, marqueses de Villafranca. Las propiedades de Villabuena pasaron, con las monjas, al monasterio de San Miguel y éste trató de recuperarlas. De hecho, en 1602, Diego de Villalobos, en nombre del monasterio de Almázcara, demandó a Pedro Osorio de Toledo, marqués de Villafranca y duque de Fernandina, por los lugares de Villabuena, Arborbuena, Quilós, los Valtuilles y otros, en los

5 Cf. Cavero Domínguez, G. y González García, M. A. 2000. El monasterio cisterciense de San Miguel de las Dueñas 197-198, León.

6 Cf. Fernández Vázquez, V. 2007. El señorío y marquesado de Villafranca del Bierzo a través de la documentación del Archivo Ducal de Medina Sidonia, p. 26 y reproducción fotográfica en documentos 8-9. Ponferrada.

7 I.C., doc. 844, datado en 1338.

8 Cf. Jiménez Suárez, M. J. 2005, doc. 205.

9 Cf. Cavero Domínguez, G. 1994, doc. 45. 
cuales el monasterio tenía 300 vasallos; lugares y vasallos habían sido incautados por los titulares del marquesado, aludiendo al aforamiento que había sido hecho por el monasterio de Villabuena a Pedro Álvarez Osorio, señor de Cabrera, Ribera y Corullón, quien después vino a ser conde de Lemos y señor de Villafranca y Ponferrada. ${ }^{10}$ Nunca el monasterio de San Miguel de las Dueñas recuperaría estos lugares ni el diploma regio por el que se certificaba su propiedad.

\subsection{San Pedro de Montes}

Podría pensarse que, al ser un monasterio femenino, era más fácilmente expoliable. Sin embargo, no fue así. También los Osorio engrosaron su patrimonio con propiedades de monasterios masculinos, como San Pedro de Montes. ${ }^{11}$ La crisis del monasterio del Oza arrancaba de fines del siglo XIII, ${ }^{12}$ y las causas estaban en las usurpaciones, como señala Herrezuelo en su decimonónica historia monástica. ${ }^{13}$ San Pedro de Montes, casa regia, ${ }^{14}$ había acudido a los monarcas $^{15}$ y a la Santa Sede, ${ }^{16}$ para proteger sus propiedades y derechos, pero los resultados no fueron los esperados $y$ no frenaron su decadencia. A comienzos del siglo XV, a la imparable crisis contribuyeron los Osorio con la desestructuración del patrimonio del otrora poderoso monasterio benedictino. Resultado del enfrentamiento entre Pedro Álvarez Osorio y los monjes del Valle del Oza fue la ocupación, por el mismo, de los lugares de Borrenes, San Juan de Paluezas, Orellán, La Chana, Valdecañada, Santalla y Rimor; el pleito entre ambos llevó al Osorio a disfrutar de los bienes en litigio, y cuando llegó a María Osorio, ésta ya había logrado reducir la renta a satisfacer al monasterio a 20 cargas de pan anual. ${ }^{17}$

10 Cf. ibídem, doc. 532.

11 Sobre este monasterio remitimos a VV. AA. 2006. San Pedro de Montes. Luz en la memoria. Actas. Ponferrada: Instituto de Estudios Bercianos.

12 En 1311, el obispo de Astorga, Alfonso, con el consentimiento del deán y cabildo, donaba varias iglesias al abad y los monjes de San Pedro de Montes, en vista de la penuria en que se hallaba y para que no fuera abandonado. La donación episcopal tenía, como contrapartida, la entrega, por parte de los monjes, de propiedades en los Barrios de Salas. El obispo parece quería ayudarles, pero engrosó su señorío de los Barrios. Cf. Cavero Domínguez, G. y González García, M. A. 2000, doc. 1.580, p. 102.

13 Cf. Archivo Diocesano de Astorga, ms. 24-10, que contiene la obra de fray Joaquín de Herrezuelo, Historia del monasterio de San Pedro de Montes, realizada en el siglo XIX; véanse especialmente los ff. 229-230. Del mismo tenor se hallan noticias en otra obra manuscrita: Archivo Diocesano de Astorga, ms. 24-11, que contiene la obra de fray Genadio Velasco, Memoria de las advertencias que conviene saber al que governare esta casa de San Pedro de Montes, especialmente en los ff. 240-243.

14 Así nombrado por Fernando II en 1162, nov. 21. Cf. Cavero Domínguez, G., Álvarez Álvarez, C. y Martín Fuertes, J. A. 2001. Colección Documental del Archivo Diocesano de Astorga, doc. 17, pp. 49-50, León.

15 Entre otras, pueden verse las confirmaciones de Sancho IV en Quintana Prieto, A. 1971., documentos 383, 384 y 385, pp. 495-501; y los diplomas otorgados, en 1379, por Juan I, en Cavero Domínguez, G., Álvarez Álvarez, C. y Martín Fuertes, J. A. 2001, documentos 112 y 113, pp. 82-83.

16 Cf. Cavero Domínguez, G., Álvarez Álvarez, C. y Martín Fuertes, J. A. 2001 , doc. 88 , pp. 135-137. Honorio IV toma bajo su protección el monasterio de San Pedro de Montes y confirma sus propiedades y derechos.

17 Cf. Franco Silva, A. 1982, 35-160.

\subsection{San Andrés de Espinareda}

La situación tampoco fue muy distinta en el caso de los monjes espinarenses, si bien parece que fue algo más tardía, en el siglo XV. De hecho, los hombres de don Rodrigo Álvarez Osorio en 1404 robaban ganado del monasterio en San Juan de la Mata. El pleito que se sucede tiene al monje Alfonso de Columbrianos como representante del monasterio de San Andrés, y, tal como testimonia el notario de Cacabelos García Fernández, se acusaba a Rodrigo Álvarez Osorio de haberse llevado cuatro bueyes. La acción era negada por el noble:

que nunca el nin sus onbres tomaron los dichos bienes... e se soubesen donde estaban que los tomasen e se algun de los defendesen que se fosen a lla justiçia desde dicho lugar que le llos entregasen.... ${ }^{18}$

Señala C. Rodríguez, en su análisis del monasterio de San Andrés de Espinareda, que, de cuantos abusos nobiliarios conocidos sufrieron los monjes espinarenses, nada es comparable a los procedentes del Conde de Lemos. ${ }^{19}$ La presencia del conde, sus atribuciones y atropellos son múltiples, y proceden de tres sectores muy conectados entre si:

- En primer lugar, de la guerra irmandiña y su proyección y extensión en la comarca berciana. A través del pleito Tavera-Fonseca, conocemos cómo fueron derribadas las fortalezas del conde de Lemos, no sólo las que poseía en territorio gallego, sino también las enclavadas en El Bierzo, entre ellas las de Sarracín, Balboa, Corullón, Cornatel y Peña Ramil/Ramiro.

- En segundo lugar, la muerte del conde de Lemos generó igualmente fuertes convulsiones sociales que afectaron al monasterio espinarense y a sus vasallos.

- En tercer lugar, hemos de referirnos a la reacción antiseñorial de 1491, que afectó espectacularmente al sector occidental berciano.

El monasterio de San Andrés mantuvo una actitud un tanto contradictoria, pero, en todo caso, como señala C. Rodríguez, era una postura de sumisión, de temor ante don Pedro Álvarez Osorio; y ello se tradujo en una respuesta difícil de comprender. Los monjes entendían que sus vasallos no debían entrar en la hermandad contra el conde buscando la ambivalencia respecto a la defensa de los intereses nobiliarios, y evitando posicionarse ante la persona de Pedro Álvarez Osorio. Su estrategia se orientaba hacia la protección frente al revanchismo que el conde pudiera desplegar contra el monasterio, sus vasallos y sus propiedades. Dicha actitud temerosa es la que se desprende, en 1469, del hecho de que se obligara al monasterio de Espinareda a aforar a un vasallo del conde unos bienes en Bembibre. El prior hace constar

que non podian escapar de lo faser aunque vian que era dampno del monasterio, con temor de los dichos señores.... e protesto el derecho del dicho monasterio por cuanto lo fasian con miedo. ${ }^{20}$

18 Jiménez Suárez, M. J. 2005, doc. 319.

19 Cf. Rodríguez González, M. C. 1992. Economía y poder en El Bierzo del siglo XV. San Andrés de Espinareda: 333: Universidad de SantiagoUniversidad de León.

20 Ídem. 
El temor fue lo que condujo a los monjes a la búsqueda de otras soluciones, es decir, a que la protección pudiera ser obtenida por otras vías. De hecho, ese mismo día el monasterio entregó la merindad de la tierra de San Andrés a Nuño González de Santalla, con la finalidad de conseguir una garantía de protección contra esos desmanes.

\subsection{Santa María de Carracedo}

Un análisis de la documentación carracetense ${ }^{21}$ nos daría una visión parecida a la registrada por los monjes espinarenses. Ya los monjes de Carracedo se habían visto obligados a solicitar de Alfonso XI una carta de amparo y defensa contra García Rodríguez de Valcarce, por haberles usurpado éste la encomienda de Carracedo, que el monarca señalaba que la había tomado para sí. ${ }^{22}$ La historiografía monástica posterior lo refleja claramente y fray Jerónimo de Llamas es muy explícito cuando escribe que estaba "muy deseoso el [García Rodríguez de Valcarce] y sus herederos de meter la mano en la hacienda de esta casa". ${ }^{23}$

Por más que los abusos fueran detectados y condenados por la monarquía, los monasterios seguían recurriendo a los mismos nobles y caballeros para solucionar sus problemas patrimoniales. En 1389, para remediar la despoblación de algunos de sus lugares, como Ambasmestas, el monasterio de Carracedo lo entregó, mediante foro, a García Rodríguez de Valcarce, su mujer e hijo, con la finalidad prioritaria de la repoblación. ${ }^{24}$ Unos años más tarde, el cillerero de Carracedo, fray Fernando Díez reclamaba a Constanza, hija de García Rodríguez de Valcarce, esposa de García de Sarmiento de Galicia, y ante Rodrigo Álvarez Osorio, hijo de aquélla, que dejara libre el lugar de Friera y los casares de Éntoma y Lagumanes. ${ }^{25}$ Pero Constanza hizo frente al monasterio y, con su hijo, se reafirmó en sus derechos sobre dichos lugares: los dos últimos realmente eran del monasterio por concesión regia.

Sin embargo, las difíciles relaciones de Carracedo serían con los Osorio leoneses. En 1378, Carracedo entregaba la encomienda de Cervantes a Álvar Pérez Osorio, señor de Villalobos. Dicha encomienda incluía los cotos de Dorna, Villalquite, Padornelo y Gestoso; a ellos se unía la quinta de Llamas y la iglesia de Cereixedo. Los monjes reclamaron a su noble encomendero defensa y amparo. ${ }^{26}$

21 Para una valoración del ámbito cisterciense masculino castellanoleonés, remitimos a los trabajos de Pérez-Embid Wamba, F. J., especialmente la obra, 1986, El Cister en Castilla y León. Monacato y dominios rurales, (siglos xii-xv), Salamanca.

22 Cf. I. C., doc. 861. Aparece sin datar, por lo que el editor del cartulario la sitúa en 1312-1350, fechas del reinado de Alfonso XI.

23 De Llamas, Jerónimo 1993. Fundación y dotación del monasterio de Carracedo, año 1593, ed. de F. González González, p. 133, Zaragoza.

24 Cf. I. C., doc. 916.

25 Cf. ibídem, doc. 974, datado en 1422

26 "...que amparedes e defendades todas las nostras cosas de quienquiera dellos quisier faser nojo e dagno, e que non consintades a ninguno ni a ninguna que pongan fueros sen demanden en los lugares del monasterio, ninguna cousa de las que non fueron acostumbradas en tiempo de los otros comenderos. E otrosy, que vos nin los encomendeyros que andaren por vos, non fagades justicia, nen pongades couto, nen demandedes iudicias nen luctuosas de los nostros sennorios". Ibídem, doc. 901.
Junto con Pedro Alfonso de Sanabria y Pedro Fernández de Bolaño, Álvar Pérez Osorio fue obligado, por ejecutoria de Juan I, en 1380, a dejar libres al monasterio de Carracedo los lugares de Cervantes, San Vicencio y Cornias. ${ }^{27}$ Todavía en 1384, el Señor de Villalobos mandaba a su encomendero en Cervantes que hiciese una pesquisa para delimitar la jurisdicción de Carracedo en los cotos de Dorna, Padornelo, Gestoso y Villaquinte. ${ }^{28}$

Las desavenencias entre Osorios, ahora con Juan Álvarez Osorio, ${ }^{29}$ y Carracedo por la tierra de Cervantes llegaron a la corte de Enrique III, cuya cancillería emitió la sentencia favorable al monasterio, al que, se señala, pertenecían los cotos de Dorna, Villaquinte, Padornelo y Gestoso. Es Ilamativo cómo Juan Álvarez Osorio alegaba, ante la corte regia, derechos familiares sobre la tierra de Cervantes, al señalar que su madre y sus antepasados por línea materna habían sido siempre señores de dicha tierra; una afirmación que no pudo probar. $^{30}$

Los Osorio no cejaban en sus reclamaciones. La iglesia de Cereixedo formaba parte del lote de la encomienda realizada en 1378. Todavía en 1419 el monasterio carracetense se veía obligado a pleitear con Pedro Álvarez Osorio por la presentación de la citada iglesia, cuya propiedad los monjes demostraron fácilmente. ${ }^{31}$

También otros nobles, con importantes conexiones familiares con los Osorio, se enfrentaron con Carracedo. Sirva de ejemplo la Casa Ducal de Benavente, cuyo titular, don Fadrique, era obligado por el monarca Enrique I a dejar libre una encomienda del monasterio de Carracedo. ${ }^{32}$

Igualmente conflictiva fue la relación carracetense con la casa de Lemos en la segunda mitad del siglo xv, especialmente con la guerra irmandiña. En primer lugar, por la usurpación y ocupación de propiedades monásticas, puestas, junto con sus rentas, al servicio de la guerra, centralizada en la fortaleza ponferradina. Las fuerzas militares del conde de Lemos obligaron a los monjes y a sus vasallos a colaborar en medidas defensivas (acarrear arena, piedra y madera, por ejemplo). La desobediencia y deslealtad eran castigadas de inmediato con la quema de imágenes, destrozos y agravios; y con la muerte, el destierro o el aprisionamiento. ${ }^{33}$ La tiranía del conde de Lemos, en el último cuarto del siglo $\mathrm{XV}$, puso a varios de los monasterios bercianos al borde de la ruina económica. A ello se unía, en el caso de Carracedo,

27 Cf. ibídem, doc. 907

28 Cf. ibídem, doc. 912.

29 Encomiendas, encomenderos y sus representantes mantuvieron una transversalidad detectable con los distintos monasterios. Así, por ejemplo, Juan Álvarez Osorio tenía como escudero a Arias Díaz de Navia, morador en Paradaseca, que tenía en arriendo varias propiedades del monasterio de San Andrés de Espinareda. Cf. Jiménez Suárez, M. J. 2005, doc. 410, datado en [1410], y doc. 413.

30 "Y que el Juan Alvarez, respondiendo havia dicho que los pedidos y jantares que llevaba en dichos cotos por titulo que para ello tenía, por quanto su madre, y por ella su padre y sus abuelos, y todos los de su linage de parte de la dicha su madre fueran sennores de Ceruantes y de tiempo inmemorial Ilebaran aquellos derechos". I. C., doc. 936, datado en 1403.

31 Cf. ibídem, doc. 965.

32 Cf. ibídem, doc. 909, datado en 1380.

33 Cf. Archivo Municipal de Camponaraya, Memorial del pleito de la Dehesa del Fabero, números 16-17. Es recogido textualmente por Balboa de Paz, J. A. 2005 (2a ed.). El monasterio de Carracedo: 72-73 León. 
otro hecho especialmente adverso: la presencia de abades comendatarios, en los que pimaron mayoritariamente intereses económicos. Tales abades comendatarios, muy frágiles y manejables, eran utilizados por el conde de Lemos para "poner y quitar" abad.

\subsection{San Miguel de las Dueñas}

La actuación de los Osorio no fue distinta de la de otros nobles respecto a los monasterios bercianos. En 1380 Juan I otorgaba carta de defensa al monasterio de San Miguel de Almázcara contra don Fadrique, duque de Benavente, que había usurpado los lugares y vasallos entregados al monasterio por la infanta Sancha, hermana de Alfonso VII, en $1152 .^{34}$

Curiosamente San Miguel de las Dueñas, al situarse en la cuenca del Boeza, un tanto alejada del sector villafranquino, no recoge, en su documentación, las injerencias que Villabuena sufrió por parte de los Osorio. Si bien ha de recordarse que asumió las reclamaciones patrimoniales heredadas del monasterio villabonense a comienzos de la época moderna.

\section{EL DESCANSO PARA LA ETERNIDAD: RELACIONES ESPIRITUALES.}

No es que las relaciones entre nobles y monjes se circunscribieran únicamente al campo económico y al funerario, y que siempre se moviesen sobre enfrentamientos y querellas. Con frecuencia parecen dotadas de un fuerte grado de cotidianeidad, o al menos asi parece desprenderse del testimonio de un anciano que señalaba haber visto a Pedro Álvarez Osorio, primer conde de Lemos, en sus años jóvenes, pernoctar en el monasterio de Santa María de Carracedo; lo describe como vestido vulgarmente y falto de modales: bebía del mismo vaso de un monje y comía lo mismo que los monjes. ${ }^{35}$

Los monasterios servían también como marco de importantes acontecimientos familiares: el citado Pedro Álvarez Osorio casó con Beatriz de Castro en 1433. Dado su parentesco, se vieron obligados a pedir las dispensas correspondientes a Roma y una vez obtenidas, refrendaron su matrimonio el 15 de septiembre de 1434, en el propio monasterio de Carracedo. ${ }^{36}$

En la vida y en la muerte, los nobles buscaron el cobijo monástico, especialmente como lugar de reposo para la eternidad. Por ello, la proyección funeraria y las cargas espirituales intensificaron dicha relación.

\subsection{La capilla de García Rodríguez de Valcarce en el monas- terio de Carracedo.}

En 1292 Sancho IV entregaba todos sus derechos sobre Toral de los Vados a García Rodríguez de Valcarce, a quien calificaba como vasallo de su propio hermano, el infante don Juan. ${ }^{37}$ García y su esposa, María Fernández, aumentaron su

34 Cf. Cavero Domínguez, G. 1994, doc. 172.

35 Cf. Franco Silva, A. 1882, p. 41.

36 Cf. Pardo de Guevara y Valdés, E. 2000. Los señores de Galicia: tenentes y condes de Lemos en la Edad Media, vol. I: 303 Santiago de Compostela.

${ }^{37}$ Cf. I. C., doc. 688 . patrimonio en la zona en los años finales de la centuria. ${ }^{38} \mathrm{~A}$ comienzos del siglo XIV, García Rodríguez de Valcarce logró la confirmación regia de Toral de los Vados por la cancillería de Fernando IV. ${ }^{39}$ Muerto García Rodríguez, hacia 1308, su viuda señalaba que ella y su marido habían entregado el lugar de Toral de los Vados al monasterio de Carracedo y a su abad, fray Juan López y a la capilla de San Cristóbal, por sus almas. Aunque podía María Fernández disfrutar durante su vida del citado lugar de Toral de los Vados, renunció a ello, y el abad y convento se comprometieron, por ello, a entregar una renta anual de $160 \mathrm{mrs}$. por San Miguel de vendimias. Pero además, la citada María entregaba otros bienes para

refasemento de la nossa capiella de sant Cristoforo sobredicha en que yo prometo mio corpo soterrar, que vos nos diestes en vosso monesterio. ${ }^{40}$

Dado que el lugar de Toral procedía de donación regia, María Fernández obtuvo la confirmación de Fernando IV por la que ella entregaba Toral de los Vados al monasterio de Carracedo. ${ }^{41}$

La capilla de San Cristóbal era, por tanto, el enterramiento inicial de los García de Valcarce, aunque no una capilla de construcción propia, sino más bien anterior. Su hijo y sucesor, del mismo nombre que su padre, García Rodríguez de Valcarce, siendo adelantado mayor de Galicia, fue quien mandó hacer la capilla -conocida con el nombre del mismoadosada a la nave septentrional de la iglesia del monasterio de Santa María de Carracedo. En 1328 mandó ser enterrado en ella, declarándose su autor, y en documento añade:

Item mando que aquellos herdamentos que eu mandey a la mia capella de Carrasedo, quellos tenga o capellan que y diser la misa e las oras; e o capellan que receba os bienes dellos e se provea dellos; e rogo al abade que le faza y decir las oras cada dia. ${ }^{42}$

Tras entregar un heredamiento en Villafranca, que había adquirido del dominico fray Gonzalo Fernández, García Rodríguez de Valcarce pidió al abad de Carracedo que mantuviera en dicha capilla un capellán que dijera misa diaria y las horas canónicas "por mia alma e de mias mulleres de que eu soy tenido, e y fincasen enterradas en la dita capella". ${ }^{43}$ Los Rodríguez de Valcarce pusieron, por ello, su escudo en Carracedo.

Esta capilla de García Rodríguez de Valcarce II es la que actualmente todavía puede verse en Carracedo. El enterramiento sobre un lucillo tiene, en su parte superior, distintos elementos heráldicos de su linaje; $y$, en la parte inferior, una inscripción recuerda el nombre de su ocupante:

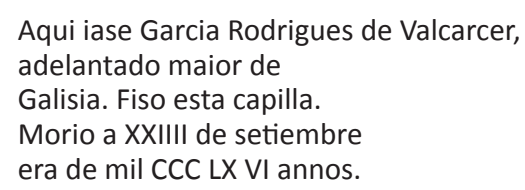

38 Cf. ibídem, doc. 706. En 1295 empeñaban propiedades de Domingo Pérez.

39 Cf. ibídem, doc. 718, fechado en 1301, Agosto, 7.

40 Ibídem, doc. 730.

41 Cf. ibídem, doc. 740.

42 Ibídem, doc. 813.

43 Ídem. 
El epitafio tiene, a su derecha, el escudo de sus titulares. Según García Lobo, el nicho de los Valcarce pertenece al siglo $x V$ y la inscripción es clasificada entre los llamados epitaphia. ${ }^{44}$

Durante los años centrales del siglo xIV García Rodríguez de Valcarce, su sucesor, no parece tuviera un buen comportamiento con Carracedo, monasterio que acudió incluso a Alfonso XI solicitando carta de amparo. ${ }^{45} \mathrm{El}$ arrepentimiento de aquél no se constata hasta su testamento, en 1364:

Item mando al monasterio de Carrasedo por emenda e satisfacemento do mal e dano que lle yo fis en a granja de Carrasedelo e en outros lugares du yo la fis, que ayan por jur de herdade, toda la herdade que eu ayo en Cubillos e que y comprou meu padre, Garcia Rodriguez. ${ }^{46}$

Arrepentido, manda ser enterrado en la capilla que hizo su padre y otorga al monasterio la heredad de Valverde, con un encargo de mil misas.

La capilla carracetense se mantuvo bajo la tutela de su sucesor, García Rodríguez de Valcarce y Balboa, y su esposa, Inés Fernández, quienes tenían su castillo en el lugar de Corullón. ${ }^{47}$ En los primeros años del siglo XV, Inés Fernández, que se decía ya viuda de García Rodríguez de Valcarce y Balboa, señalaba el interés de su marido por entregar la tierra de Friera y los casares de Éntoma y Lugamanes, que retenían por la fuerza, al monasterio carracetense. ${ }^{48}$ Por eso, ella renunciaba a sus derechos, para descarga del alma de su marido, y favorecía la toma de posesión de dichos lugares por los monjes carrecetenses. ${ }^{49}$

Todavía unos años más tarde, la citada Inés Fernández, que vivía en el castillo de Corullón, mandó a su sobrino y procurador en Villafranca, Vasco Pérez, que gestionase el aforamiento de la tierra de Friera y los lugares de Éntoma y Lugamanes, que el monasterio le había otorgado a cambio de la correspondiente renta anual. ${ }^{50}$

Fallecida Inés Fernández y, probablemente, enterrada en la capilla carracetense, el monasterio dejó de ser lugar preferente y atractivo. Constanza, hija de García Rodríguez de Valcarce y Balboa y de Inés Fernández, casó, en primeras nupcias, con Pedro Álvarez Osorio, primer señor de Cabrera y Rivera, de quien tuvo un hijo, llamado Rodrigo Álvarez Osorio; y, en segundas nupcias, con el adelantado de Galicia, García Sarmiento. Cuando, en 1422, los monjes de Carracedo le solicitaron que dejara libre la tierra de Friera y los lugares de Éntoma y Lugamanes, ella y su hijo, desde el castillo de Corullón, se enfrentaron al monasterio defendiendo su propiedad. Lejos de avenirse, Constanza y Rodrigo Álvarez Osorio se reafirmaron en sus derechos sobre Friera, Éntoma y Lugamanes y no reconocieron que se hubiera hecho ninguna donación por Inés Fernández. ${ }^{51}$

44 García Lobo, V. 1997. “De epigrafía cisterciense. Las inscripciones del monasterio de Carracedo", Cistercium 208: 189-205. Del mismo autor puede verse también: García Lobo, V. 1996. "La epigrafía en Carracedo", en Monasterio de Santa María de Carracedo: 67-68 León.

45 Cf. I. C., doc. 861. Aparece sin datar, pero se sitúa hacia 1329.

46 Ibídem, doc. 884.

47 En 1399, Inés, en nombre suyo y de su marido, estando en la citada capilla, realizó una permuta con el monasterio de Carracedo sobre unas casas en Cacabelos. Cf. ibídem, doc. 934.

48 Cf. ibídem, doc. 956, datado en 1412.

49 Cf. ibídem, doc. 957.

50 Cf. ibídem, documentos 971 y 972, datados en 1422.

51 Cf. ibídem, doc. 975.
Pedro Álvarez Osorio tenía su capilla funeraria en la iglesia de los dominicos de Benavente, donde fue enterrado junto con su mujer, Constanza García de Valcarce. ${ }^{52}$ De alguna manera, Constanza olvidaba su interés cisterciense y se volcaba en los mendicantes.

Los García de Valcarce acabaron por abusar de la hospitalidad funeraria de los monjes carracetenses; ampliaron su espacio funerario, ocupando la nave norte de la iglesia; y provocaron que, en el siglo XVI, el monasterio los desposeyese de sus derechos funerarios. ${ }^{53}$

\subsection{El enterramiento en San Francisco}

Ya desde comienzos del siglo $\mathrm{xV}$, el atractivo religioso de que disponían los frailes hizo que los García de Valcarce perdieran su interés por su monacal capilla carracetense para buscar cobijo funerario en la iglesia conventual de San Francisco de Villafranca. En 1406 García Rodríguez de Valcarce y su esposa, Inés Fernández, compraron al citado monasterio de San Francisco de Villafranca la capilla de San Salvador, por 7.000 mrs. ${ }^{54}$ Los franciscanos utilizarían la citada cantidad para pagar "los órganos" que habían adquirido recientemente y para su mantenimiento. Eran 7.000 mrs. que no excluían la correspondiente dotación de la capilla que previamente se había hecho y que incluía unas rentas de diez miedros de vino y cinco modios de pan. La capilla franciscana de San Salvador sería mantenida como lugar de enterramiento por los Osorio.

Realmente el protagonismo franciscano villafranquino se inició en el siglo XIV al morir María de la Cerda, nieta de Alfonso $X$, que solicitó ser enterrada en el citado convento franciscano. $^{55}$ En segundo lugar, estaba la concesión de 100.000 mrs. otorgados, en 1400, por el poderoso personaje gallego Pedro Enríquez, conde de Trastámara, señor de Villafranca desde 1394, por concesión de Enrique III. Pedro Enríquez

52 En la capilla mayor de los dominicos benaventanos estaban enterrados al menos los padres, Álvar Rodríguez Osorio y María Fernández de Sandoval, y el abuelo, Juan Álvarez Osorio, de Pedro Álvarez Osorio, señor de Cabrera y de Rivera; allí serían enterrados él y su mujer, Constanza García de Valcárcel. Es de gran interés el epitafio que se hallaba colocado sobre la tumba de María Fernández de Sandoval: "ESTA CAPILLA MANDO FACER DON PEDRO OSORIO, CONDE DE LEMOS, SEÑOR DE CABRERA E RIVERA E VILLAFRANCA, E PONFERRADA, A SERVICIO DE DIOS E DE LOS NOBLES CAVALLEROS, SUS ABUELOS, QUE EN ELLA ESTAN SEPULTADOS". Pueden verse al respecto las notas aclaratorias de González González, F. 1993, 134-135.

53 Pedro de Toledo, casado con María Osorio Pimentel (ambos, marqueses de Villafranca), acabó por reclamar los bienes que los García de Valcarce habían entregado a Carracedo para la capilla; su reclamación (con el robo de escrituras) era hecha por los derechos de María Osorio. "El monasterio reacciona a tal expolio prohibiendo los enterramientos de los García Rodríguez de Valcarce y quitando las sepulturas de una capilla que quedaba ya sin dotación... Llamas será testigo excepcional del postrer coletazo expurgador, cuando, en 1583, ya él monje de aquel cenobio, vio desaparecer el último sepulcro de un biznieto homónimo de García Rodríguez de Valcarce II, quien con su innominada mujer yacía en un lucillo, a la entrada de la capilla de San Miguel, en un sarcófago sobre leones". González González, F., 1993: 136.

54 Cf. Gómez Bajo, M. C. 1994. Fondo documental del priorato de Santa María de Cluny de Villafranca del Bierzo, siglos XIV-XV, doc. 11. Ponferrada.

55 Gancedo Sandes, D. 2001. "El espacio urbano de Villafranca", Revista del Instituto de Estudios Bercianos, 27: 270. 
fue el padre de Beatriz de Castro, que después se convertiría en esposa, la primera, de Pedro Álvarez Osorio I. Pedro Enríquez murió en Orense, y en su testamento, otorgado el 29 de abril, mandó que sus restos descansasen eternamente en el monasterio de San Francisco de Villafranca. Enríquez y Osorio, fundidos en la casa condal de Lemos a partir de 1430, fueron los protagonistas del lugar de enterramiento de los franciscanos villafranquinos así como protagonistas de su crecimiento económico, que se tradujo en la ampliación del recinto conventual y en la mejora de su templo.

Por otro lado, al ser elegido lugar funerario preeminente por el primer conde de Lemos, algunos miembros de su familia optaron por el mismo destino final. Es el caso de García Enríquez Osorio, que fue a arzobispo de Sevilla en los años 1442-1448. Al final de sus días, éste decidió retirarse a los franciscanos bercianos. Como señala C. Álvarez, su contribución se tradujo en la reedificación de la capilla mayor y la construcción de otras dependencias. ${ }^{56}$ Por supuesto, a ello debe añadirse la construcción de su sepulcro que así fue descrito:

Como el Arzobispo edificó la capilla mayor para que fuere entierro de los señores de la Casa, luego que llegó la ocasión pusieron sus sepulcros lebantados en medio della. Son tres tan contiguos que a la primera vista representan uno cuadrado; son todos de márbol de estas montañas, con figuras lindísimas de más que de medio relieve y de la vida y muerte de Christo, de Su Madre y de San Francisco. ${ }^{57}$

El fallecimiento de Beatriz de Castro sucedió en la primavera del año 1455, el 10 de abril, y sus restos fueron también depositados en el monasterio franciscano, en el panteón familiar, a mano derecha; ${ }^{58}$ la descripción de su sepulcro es elocuente:

con sola una toca, vien ceñida al rostro, presa ligeramente devajo de las barbas, y un rosario al cuello enlazado con su grazia sobre el pecho; basquiña y ropa tan largas que hazen faldra, si bien por delante descubren unos zuecos....; tiene bestido sobre todo un baquero no mas largo que hasta la rodilla, cerrado y mui plegado. Por los golpes de el saca los brazos con mangas justas sobre el codo, pero anchas y pegadas a los ombros; tienen las manos unas horas grandes o berviario, y en el sepulcro el epitafio siguiente: Aquí jaze doña Beatriz, señora de Villafranca, Ponferrada e Monforte e de Caldelas. Fue casada con don Pedro Osorio, conde de Lemos, señor de Cabrera e Rivera. ${ }^{59}$

56 Cf. Álvarez Álvarez, C. 1995. "Mecenazgo nobiliario y órdenes mendicantes en la diócesis de Astorga (1250-1500)", en El Monacato en la diócesis de Astorga durante la Edad Media, Actas del Congreso: 117144. Astorga.

57 Biblioteca Nacional (=BN), ms. 19.418, que contiene la Chrononologia de los Ilustrisimos Jueces de Castilla, Nuño Peres Pesura y Layn Calvo; antecesores de la esclarecida familia de Castro; condes de Andrade, Lemos y Villava y marqueses de Sarria; de cuyos progenitores y sucesores por lineas derechas y transversales se tratan en este, fol. 369r. Se trata de la obra de fray Malaquías de la Vega, considerada historia oficial de la casa de Lemos, que fue hecha a petición de Pedro de Castro, VII conde de Lemos. Cit. Pardo de Guevara y Valdés, E. 2000., p. 411.

58 Una pequeña biografía ha sido publicada por García Tato, I., 2008. Doña Beatriz de Castro, Ponferrada.

59 Así recoge sus armas: "por el medio, en la parte superior y derecha un castillo, a la yzquierda un leon rampante, y en la parte baxa seis roeles de tres en tres, que son las armas de los Castro y Enríquez... seis roeles con los castillos y leones, por dezender del rey don Alonso el último". Ibídem, nota 1097.
Sobre su sepulcro está la heráldica de su casa: Castro y Enríquez.

Fray Malaquías, genealogista de la Casa de Lemos, pone de relieve el aprecio de Pedro Álvarez Osorio por doña Beatriz:

... en todos los edifiçios que haçia puso el nombre de la condesa doña Beatriz de Castro y en los epitaphios de las sepulturas suyas y de su hijo, diciendo que fue señora de Lemos y Sarria, Caldelas, Villafranca y Ponferrada y otras villas y señoríos, por herencia de su hermano el duque de Arjona y de sus padres y abuelos. ${ }^{60}$

En 1457 moría doña María, segunda hija de Pedro Álvarez Osorio y Beatriz de Castro, y también era enterrada en el citado panteón franciscano berciano; en la Relación de los sepulcros de la Casa de Lemos se describe su lauda en los siguientes términos:

Al pie del gran sepulcro, al lado de doña Beatriz, pero en el suelo, está una buena lápida de mármol que tiene gravados dos escudos en los ángulos de arriba con un letrero a la redonda. En el primer escudo los dos lobos andantes de los Osorio, y en el segundo el castillo, el león y seis roeles de los Castro de Lemos, y el letrero dice: Aqui jaze doña María Osorio, fija de don Pedro Osorio, conde de Lemos, señor de Cabrera e Rivera, e doña Beatriz, su mujer. Fallezió de edad de onze años, sacados dos dias de septiembre, año MCCCCLVII. ${ }^{61}$

En 1467, tras otorgar su testamento, moría don Alonso, primogénito y único hijo de los condes de Lemos, siendo enterrado en la capilla mayor de la iglesia conventual villafranquina de San Francisco, ${ }^{62}$ donde descansaban también los restos de sus padres y algunos de los hermanos del primer conde de Lemos, como señala fray Malaquías. ${ }^{63}$ El sepulcro de don Alonso, de menor entidad, estaba

a la mano izquierda de don Pedro Alvarez Osorio ...., y al parezer de caballero mozo armado y con la espada en la mano tendida desde el pecho a los pies, frente de ellos y en lo baso del sepulcro se ve la mitad superior de un escudo, que la otra metad baxa esta enterrada: tiene a la mano derecha un castillo y en la izquierda un solo lobo andante... Está en este sepulcro otro epitafio cavado en la piedra, como los demas, que dice: Aqui jase don Alonso, fijo de don Pedro Osorio, conde de Lemos, señor de Cabrera y Ribera, e de doña Beatriz, su muger. Vivió veinte e cinco años, murió en año del Nascimiento de Nuestro Señor lhesu Christo de mill e quatrozientos e sesenta e siete años. ${ }^{64}$

Octogenario murió Pedro Álvarez Osorio, en 1483, después de haber enfermado gravemente en su castillo de Corullón, y fue enterrado en el mismo panteón familiar, al

60 BN, ms. 19.418, fol. 348v, en el que se contiene la Chronología de los Ilustrísimos Jueces de Castilla, Nuño Peres Pesura y Layn Calvo; antecesores de la esclarecida familia de Castro; condes de Andrade, Lemos y Villava y marqueses de Sarria, de fray Malaquías de la Vega. Lo recoge Fernández Vázquez, V. 2007, pp. 38 y 40.

61 Archivo Ducal de Alba, Sección de Lemos, C-228-29. Cit. Pardo de Guevara y Valdés, E., 2000, p. 413.

62 Cf. Gancedo Sandes, D. 2001, pp. 5-68.

63 Cf. BN, ms. 19.418, fol. 362v. Lo cita Fernández Vázquez, V. 2007, p. 40.

64 Archivo Diocesano de Astorga, Sección de Lemos, C-228-29. Cit. Pardo de Guevara y Valdés, E., 2000, pp. 413-414. 
lado de Beatriz de Castro y sus hijos María y Alonso. En su sepulcro

un bulto de cavallero armado con su montante: saio a lo antiguo y una gora de faldas largas, lebantadas por las espaldas y la frente y partidas por los lados, que todo el representa gran authoridad y señorio, si bien tiene el rostro sin barbas y lampiño. ${ }^{65}$

A sus pies se encuentra la heráldica de su linaje, los lobos de los Osorio, y la inscripción con su nombre y los títulos: conde de Lemos, señor de Cabrera y de Ribera.

El espléndido y generoso mecenazgo que el conde de Lemos y sus hermanos realizaron con San Francisco, se prolongó, según C. Álvarez, en el último cuarto del siglo XV con Juana Osorio, hija del conde. El enfrentamiento de los descendientes de Pedro Álvarez Osorio generó una disposición salomónica por parte de los Reyes Católicos, quienes, en compensación por la pérdida del título condal de Lemos, concederían a Juana Osorio el título de marquesa de Villafranca. Para salvaguardar sus intereses, poco tiempo después, sería concertado su matrimonio con Luis Pimentel, segundogénito del conde de Benavente. Ambos serían los primeros marqueses de Villafranca y ejercerían una continuada labor de patronato sobre el convento franciscano, el más importante de la villa, cabeza de su señorío, al que convirtieron en panteón familiar de su linaje y al que periódicamente entregaron abundantes bienes. ${ }^{66}$

Sin embargo, Luis Pimentel, fallecido en 1497 en Alcalá de Henares, sería conducido, por decisión paterna, a los franciscanos recoletos de Villalón, en cuya capilla funeraria, situada en la capilla mayor, se hallaban también los restos de su madre. Medio siglo después serían llevados los restos de ambos a su capilla funeraria de los franciscanos de Benavente. ${ }^{67}$

La primera marquesa fundó la capilla de la Encarnación, dotándola con treinta mil mrs., ochenta cargas de centeno, siete modios de trigo y dos mil mrs. de renta y juro anuales situados en el portazgo de la villa. En ella descansarían también los restos de doña María Osorio Pimentel, segunda marquesa, a su muerte, en 1539. Cuando algunos años después, en 1555, hizo testamento el marqués Pedro Álvarez de Toledo, su marido, recogió igualmente cargas espirituales: señalaba que deberían decirse por su alma diez mil misas, mitad en Italia y mitad en Villafranca. En su villa berciana, el virrey de Nápoles distribuyó sus misas entre la colegiata de Santa María, el monasterio de San Francisco de Villafranca y el convento franciscano de Cabeza de Alba. ${ }^{68}$

65 Así lo recoge fray Malaquías, 1993, fol. 370. Cit. Pardo de Guevara y Valdés, E., 2000, p. 411.

66 Cf. Álvarez Álvarez, C. 1995, p. 128.

67 Información completa sobre la capilla sepulcral de Villalón y el retrato funerario de don Luis Pimentel, puede verse en Fernández Vázquez, V. 2007, p. 47 y, especialmente, la nota 23bis. En la p. 46 se recoge un detalle del retrato funerario de Luis Pimentel, primer marqués de Villafranca.

68 "Ytem mando que por la mi anima y por la de la marquesa mi muger que santa gloria aya y por los defunctos de entramos a dos, se digan diez mil misas, las cinco mil en estas partes y las otras cinco mil en mi villa de Villafranca y en Cabeça de Alua, a saber: dos mil missas en la yglesia mayor de Villafranca y dos mil en sanct Francisco y mil en Cabeça de Alua, por las quales misas se dara la limosna lo acostumbrado, y las missas de acca las haran dezir mis testamentarios donde a ellos mejor paresçera. Ytem mas porque yo por el anima de la marquesa mi muger
Los segundos marqueses villafranquinos, cuya vida transcurrió durante años en Italia, crearon también su monumento funerario en la iglesia napolitana de Santiago de los Españoles como virreyes de Nápoles. ${ }^{69}$

\subsection{La creación de nuevos espacios conventuales}

Aunque los Osorio villafranquinos fundaron en su villa un convento femenino, llamado de la Concepción, nunca lo utilizaron como lugar de enterramiento, tal vez debido a las muchas dificultades económicas que sufrió el convento en su primer siglo de existencia. Aunque fuera ya de nuestro ámbito cronológico medieval, una nueva fundación, también femenina y clarisa, por parte de los titulares del marquesado, se convirtió en referente funerario en época moderna. Es el convento de la Anunciada, fundado por el marqués don Pedro de Toledo para su hija María, en 1604, bajo la tutela vicarial de los franciscanos recoletos de Cabeza de Alba. ${ }^{70}$ Esta nueva fundación y, especialmente, el abadiato de María de Toledo y Mendoza, en religión Sor María de la Trinidad, acabaron por hacer de las clarisas villafranquinas un lugar apropiado para el enterramiento del linaje Osorio de los marqueses en el siglo xvii.

\section{EL APELLIDO OSORIO ENTRE LOS MONJES}

que santa gloria aya tengo ordenado que se cante cada dia una missa y se diga otra rezada en la yglesia mayor de mi villa de Villafranca, por las quales se pagan quarenta mil mrs., a saber trenta mil por la cantada y diez mil por la rezada mando y es mi voluntad que esto perpetuamente se diga assi como esta ordenado. Yten mando que otra misa que yo hago dezir en el monasterio de nuestra señora de Cabeça Dalua tanbien por descargo del anima de la marquesa mi muger, se diga perpetuamente $y$ se continue cada dia como se dize, y por ella se de al sindico de los dichos frayles diez mil mrs. para reparacion de la dicha casa, y vestir los frayles y otras necesidades. Y porque yo despues de la muerte de la virreyna mi muger, que santa gloria aya, de mis propios bienes compre trezientos mil mrs. de juros perpetuos para dar a la yglesia de Sanctiago por el derecho y action que pretendia y tenia a la villa de Cacabelos, por tanto para cumplimiento destas tres misas que mando dezir cada dia del año perpetuamente en Villafranca, dos en la yglesia mayor y en Cabeça Dalua la otra quiero que se paguen estas tres misas en los diezmos de la dicha villa de Cacabelos y no quiriendolos pagar mis herederos alli seran obligados a pagar los dichos cinquenta mil mrs. en otra qualquier parte que ellos querran.... Ytem por quanto yo y la marquesa que aya gloria, ordenamos para descargo de nuestras conçiençias que se diesen quarenta hanegas de trigo a los frailes de Cabeça Dalua quiero que ni mas ni menos de aqui adelante perpetuamente esto se les de de limosna como agora yo tengo mandado que se haga y creo se haze. Ytem que las misas que en San Francisco de Villafranca se dizen, que la marquesa mi señora y suegra dexo y la cera y oferta de los santos, se continue como hasta ahora, y mando y ordeno que al dicho conuento se le den cada año perpetuamente sesenta hanegas de trigo". Archivo del monasterio de Nuestra Señora de la Concepción, año 1555 (Universis et singulis...), pergamino, sin catalogar.

69 Una reproducción puede verse en Fernández Vázquez, V. 2007, p. 51.

70 María había profesado previamente en el monasterio de la Concepción, pero insistió tanto en la fundación del convento de la Laura, que terminó por involucrar al marqués en una nueva fundación femenina y villafranquina. Cf. Arias, M. C. 1994. "Doña María de Toledo y su obra", en Las Clarisas en España y Portugal, Congreso Internacional, Salamanca, 20-25 de septiembre de 1993, vol. I: 343-378. Madrid. 
Escasa presencia del apellido Osorio hallamos en los monasterios bercianos; sin embargo, en algunos casos, es bien señalada y los protagonistas ocuparon cargos abaciales.

\subsection{Carracedo y los Osorio, abades comendatarios}

El abadologio de Carracedo recoge a varios abades comendatarios, especialmente desde el último cuarto del siglo XIV. El primero de ellos, citado por el padre Yepes, ${ }^{71}$ es Álvaro Osorio, comendatario (¿ं?) entre 1375 y 1395 . Su presencia no se detecta en la documentación y tampoco hemos obtenido datos sobre su procedencia, tal vez ésta sea la razón de los interrogantes que el padre Yepes le adjunta.

Hijo del conde de Trastámara y hermano del primer marqués de Astorga, otro Osorio, Luis, acumuló y desempeñó varias prebendas eclesiásticas; entre otras, la de abad comendatario de Carracedo, entre 1471 y $1474 .{ }^{72}$ En primer lugar, fue canónigo y administrador de la sede metropolitana de Santiago de Compostela, desde donde defendió los intereses paternos en Galicia. Posteriormente sería deán de la iglesia de León, arcediano del Páramo en la iglesia de Astorga, presidente de la Chancillería de Valladolid y obispo de Jaén. La figura de Luis Osorio, estudiada por Martín Fuertes, presenta este perfil:

personaje inquieto y ambicioso, frecuentemente envuelto en litigios, afortunado por lo general en sus empresas, su esbozo biográfico ofrece un especial atractivo porque, en una época protomoderna, su figura encarna un acabado modelo de caballero-prelado, componente indispensable para la comprensión de la superestructura político-militar que presidía la sociedad hispánica del Medioevo. ${ }^{73}$

Con su experiencia en la sede compostelana, la presencia de Luis Osorio en Carracedo como comendatario se tradujo en difíciles relaciones y en una rapacidad económica insospechada. En primer lugar, en 1471 autorizó a Juan de Canedo, prior de la granja de Dorna, a realizar foros y arrendamientos. ${ }^{74} \mathrm{Al}$ año siguiente, don Lope, su abad, renunció a la abadía de Oscos, jurisdicción de Carracedo; y Luis Osorio aceptó la renuncia. ${ }^{75} \mathrm{El}$ de Oscos aludía a su vejez como motivo fundamental para la renuncia, pero el desacuerdo de la abadía filial respecto a la actuación del abad comendatario carracetense estaba detrás de la resignación. El problema más crítico procedía de la no aceptación del abad comendatario por una parte de la comunidad conventual carracetense, cuyo malestar era manifiesto desde el comienzo pero que trascendió el año 1472, al ser elegido otro abad, Alonso de Soto, que pertenecía al grupo inconformista. Luis Osorio apeló ante las jerarquías cistercienses y denunció los hechos con el fin de recuperar la autoridad.

71 Cf. Yepes, A. 1617. Coronica de la orden de San Benito, vol. III: 435. Valladolid.

72 Cf. De Llamas, Jerónimo, 1993, pp. 204-205. El editor recoge, a pie de página, las listas comparadas elaboradas por el padre Yepes, Emilio José Prieto y las escasas recogidas por Jerónimo de Llamas.

73 Martín Fuertes, J. A. 1979. "Don Luis Osorio, caballero y prelado leonés del siglo xv. Esbozo biográfico", Estudios humanísticos, 1: 109120; especial para el texto recogido es la p. 109.

74 Cf. I.C., doc. 1.138.

75 Cf. Ibídem, documentos 1.143 y 1.144 .
Los cistercienses delegaron en el abad del monasterio leonés de Santa María de Nogales, quien, como juez, dictaminó a favor de Luis Osorio. ${ }^{76}$

Que el dictamen no había sido aceptado quedó bien claro dos años más tarde, cuando los monjes de Carracedo lograron que el pontífice Sixto IV atendiera sus reivindicaciones contra el abad comendatario, don Luis Osorio. El pontífice nombró al abad de Espinareda y al prior de Santa María de Cluny de Villafranca para que siguieran la causa. ${ }^{77}$ Mientras tanto, al frente de la comunidad figuraba Alfonso de Soto; lo que evidencia que Roma dictaminó a su favor. De hecho, estaría al frente de Carracedo al menos de 1474 a 1482 .

Agotada la vía eclesiástica, Luis Osorio recurrió, en 1480, a la justicia ordinaria, presentando las pruebas para demostrar sus derechos a la abadía carracetense. ${ }^{78}$ Nunca más volvió a ella. Los abades comendatarios, sin embargo, continuaron despojando al monasterio y situándolo al borde de la crisis.

\subsection{La presencia Osorio entre las monjas}

La tardía presencia de los Osorio se dejó sentir también en los monasterios femeninos. Urraca Osorio era abadesa en San Miguel de las Dueñas en $1474 .{ }^{79}$ Con todo, es casi una excepción en este monasterio, y tampoco tuvieron presencia en el monasterio de Villabuena, que desapareció al ser derruida la fábrica monástica, trasladándose la comunidad al de San Miguel de las Dueñas, formando una única comunidad desde el primer cuarto del siglo XVI. ${ }^{80}$

Los modernos conventos franciscanos femeninos de Villafranca acogieron a los miembros femeninos del linaje Osorio desde el segundo tercio del siglo XVI, estando escasamente representados en los monasterios cistercienses. En el convento de la Concepción se encuentra, entre las abadesas, el apellido Pimentel, de la casa de Benavente, con la que enlazó el linaje villafranquino de los Toledo y los Alba y de otras casas nobiliarias con las que los marqueses villafranquinos estaban emparentados. ${ }^{81}$

De hecho, mientras que en el caso de la fundación de la Concepción no parece haber un interés primario por crear

76 Cf. Balboa de Paz, J. A. 2005, pp. 76-77.

77 Cf. I. C., doc. 1.149.

78 Cf. Archivo General de Simancas, Registro General del Sello, 1480, ff. 95

79 Cf. Cavero Domínguez, G. 1994 doc. 182. Otra abadesa, doña Antonia Osorio, es mencionada en la documentación de Almázcara, pero ya es de fines del siglo XVIII; véase ibídem, documentos 1.581 y ss., en los años 1792-1794.

80 Sobre la crisis del monacato cisterciense femenino en El Bierzo, remitimos a nuestro trabajo Cavero Domínguez, G. 1992. "El monasterio cisterciense de San Miguel de las Dueñas. La crisis del siglo XV", Actas del Congreso Internacional sobre San Bernardo e o Císter en Galicia e Portugal, vol. II: 733-744. Ourense.

81 Juana Osorio, primera marquesa de Villafranca, se casó con Luis Pimentel, de la Casa de Benavente. Su hija y heredera sería María Osorio Pimentel, segunda marquesa de Villafranca, que a su vez contraería matrimonio con Pedro Álvarez de Toledo, perteneciente a la Casa de Alba; esta segunda unión supuso la formación del poderoso dominio de los Osorio-Toledo. Véase Cavero Domínguez, G. 1990. “Fundaciones concepcionistas bercianas (siglo XVI)", en La orden concepcionista. Actas del I Congreso Internacional, vol. I: 411-426, particularmente pp. 412413. León. 
un convento para el acceso de algún miembro del linaje Osorio, en el caso de la posterior fundación del convento de la Anunciada, a que ya nos hemos referido, la documentación señala expresamente su creación para doña María de Toledo y Mendoza, hija del marqués.

\section{LA FUNDACIÓN DE CONVENTOS}

Los franciscanos se habían asentado en Villafranca en un magno escenario, próximo a la iglesia de Santiago, en las inmediaciones de la ruta jacobea, ya en la primera mitad del siglo XIII. 82

Pero el noroeste berciano contaría con otra fundación franciscana impulsada por los Osorio. Inicialmente se trató de un eremitorio y se situó en Cabeza de Alba, al menos desde el primer cuarto del siglo XV. En los años centrales de dicha centuria, esta fundación eremítica se integró en la "Observancia". Hacia 1440, bajo la tutela de Pedro Álvarez Osorio, señor de Cabrera y Ribera, posteriormente conde de Lemos, nacería el convento, con la transformación del eremitorio. Además, el conde de Lemos, como patrono generoso, solicitaría la bendición pontifical de Eugenio IV, de cuya cancillería procedería la bula confirmatoria. ${ }^{83}$

Aunque ya en época tardía, a comienzos del siglo XVI, los Osorio también participaron en la fundación de nuevos conventos femeninos y adquirieron cierto compromiso con la nueva espiritualidad mendicante, alejándose, sin duda, del ahora poco atractivo y muy decadente Císter.

Los segundos marqueses de Villafranca, María Osorio Pimentel y Pedro Álvarez de Toledo, fundaron el convento de la Concepción de Villafranca. Efectivamente la fundación ya estaba realizada cuando, en 1539, murió la marquesa. Se edificó en un lugar relativamente secundario, entre los dos ríos, el Burbia y el Valcarce, en el llamado barrio de Tejedores, al lado de la ruta jacobea a su paso por la villa. La pronta desaparición de la marquesa y el alejamiento de Pedro Álvarez de Toledo, virrey de Nápoles, que vivió fuera de los estados del marquesado, complicaron la puesta en marcha de la nueva fundación, que arrastró cierta penuria a lo largo de todo el siglo xvı. ${ }^{84}$

La nueva fundación, según señala el testamento de Pedro Álvarez de Toledo, estaría bajo "la orden de San Francisco, e devajo de la obediencia de dichos frayles". Su interés era dotar a su villa marquesal con un convento franciscano femenino bajo la tutela del convento de San Francisco de Villafranca. Sin embargo, a comienzos del siglo XVI, la espiritualidad femenina se inclinaba, de forma espectacular, hacia la espiritualidad de Beatriz de Silva, fundadora de la Orden Concepcionista, en cuya línea concepcionista acabaría por enmarcarse la fundación de los marqueses villafranquinos.

La dotación económica inicial era de 64.000 mrs. (8.000 por cada monja); una dotación, a priori, suficiente, pero muy controvertida puesto que sería impugnada por el tercer marqués, que no aceptaba el testamento paterno, realizado sobre los bienes del patrimonio de María Osorio.

82 Cf. Gancedo Sandes, D. 1995. "El convento de San Francisco de Villafranca del Bierzo. Siglo XV", en El Monacato en la diócesis de Astorga durante la Edad Media, Actas del Congreso: 269-275. Astorga.

83 Cf. Álvarez Álvarez, C. 1995, p. 133.

84 Cf. Cavero Domínguez, G. 1989, especialmente pp. 416-417 y 424-425.
El convento era, ya desde la fundación, de reducidas dimensiones y para una comunidad de sólo ocho monjas dotadas, con un expreso control del acceso al claustro por parte del linaje Osorio. Las monjas, al acceder al convento, debían aportar sus ropas de vestir y de cama, libros y enseres (alhajas). ${ }^{85}$

Cuando, en 1549, accedieron al claustro Marina y Catalina de Guevara, hijas del gobernador del marquesado villafranquino, Gonzalo de Valcarce, y de su mujer, Ana Becerra, se señala expresamente que la abadesa del monasterio era una Pimentel, de nombre doña Francisca. ${ }^{86}$ Ambas entraban en calidad de monjas con dote; para ellas entregaba su padre diez cargas de pan de renta, cinco de trigo y cinco de centeno, más dos puercos cebados o dos ducados. Las propiedades sobre las que se sustentaba dicha renta se hallaban en distintos lugares del entorno, desde Dragonte a Corullón, Villagroy, Valtuille de Arriba y Villadecanes.

Sin embargo, antes de que muriese el virrey, en 1551, una bula de la cancillería de Julio III autorizaba una dotación de 30 monjas de coro, más cinco o seis legas o sirvientas. Se expresaba que las monjas procediesen especialmente del reino de Galicia, y de forma más concreta de la diócesis de Astorga. Se expresaba que, si aumentaba el número de monjas, los marqueses podían elegir hasta seis religiosas conventuales -que después acabarían siendo únicamente cuatro-y que no necesitarían entregar dote. ${ }^{87}$

Para ello la dotación económica fue elevada a cien mil mrs.: una dotación desproporcionada, en la simple relación de 8 a 30, y más insuficiente si se atiende a otro problema realmente serio, arrastrado desde los primeros momentos de la fundación y al que ahora se hacía alusión. Se trata de la dotación hídrica: el nuevo convento habría de tomar el agua en Dragonte, pequeño pueblo al Oeste de Villafranca, situado entre el Burbia y el Varcálcel; el punto de toma sería una fuente. La canalización no se había llevado a cabo aún a fines del siglo XVI. La dificultad de su conducción hizo que finalmente el agua fuese tomada en Puente del Rey, al Norte de Villafranca, donde al puerto en que se tomaba el agua se le conocía con el nombre de "avanzado de las monjas". Pedro Álvarez de Toledo era consciente del problema de la canalización del agua y de que no se habían terminado las obras de fábrica; por lo que mandó que los cien mil mrs. fueran entregados, "al menos", mientras no se solucionaran ambos problemas; a ello aluden las monjas, ante Felipe II, en cuya real ejecutoria se señala:

... deçimos que el virrey don Pedro de Toledo, marques que fue deste marquesado e bisorrey de Napoles en su testamento con que murio, entre otras cosas que en el mando y hordeno, dexo mandado y hordenado que diesen a esta sancta cassa cada anno çien mill mrs. en quanto no se acabase de haçer e se traxese a ella el agua que se conçerto traher e dexolos sennalados en çiertas partes en las dichas clausulas declaradas e dexo al conde don Alfonso de Benabente por su testamentario e complidor de las mandas e legatos...88

85 Franco Silva, A. 1982, p. 124. Cita el Archivo Ducal de Medina Sidonia, leg. 5.046 .

86 Cf. Archivo de la Concepción de Villafranca, s. c., datado en 1549, enero, 28, Villafranca.

87 Cf. Franco Silva, A. 1982, p. 124; remite al Archivo Ducal de Medina Sidonia, leg. 5.046.

${ }^{88}$ Archivo de la Concepción de Villafranca, Real Ejecutoria de Felipe II, cuaderno en papel, forrado en pergamino. 
La escalera monumental, con ricos esgrafiados, que se halla en el interior del convento está flanqueada por los escudos de los fundadores: el de los Osorio de doña María y el de los Álvarez de Toledo de don Pedro. ${ }^{89}$

A modo de conclusión, podemos afirmar que los monasterios bercianos sufrieron la injerencia nobiliaria que los arrastró a una fuerte crisis económica en los siglos XIV-XV. Con frecuencia la entrega de propiedades en encomienda permitió el trasvase de parte de la hacienda monástica a patrimonios nobiliarios, lo que explica que varios diplomas (algunos fundacionales y otros dotacionales) se encuentren en archivos nobiliarios (Casa de Alba, Casa de Medina Sidonia).

Por otro lado, la presencia de abades comendatarios, a veces también procedentes del linaje Osorio, contribuyó definitivamente a la crisis monástica bajomedieval del monacato berciano.

Cistercienses y mendicantes fueron los elegidos para fundar las capillas funerarias de los Osorio y de sus linajes precedentes y posteriores. Las iglesias de Santa María de Carracedo y San Francisco de Villafranca serían los lugares preferidos para el descanso funerario del linaje fundador del marquesado villafranquino. Del éxito mendicante se deduce el gran interés por la fundación de un panteón familiar en el monasterio de San Francisco de Villafranca, en el que reposaron los Osorio anteriores a la creación del marquesado villafranquino, bajo el mecenazgo de la casa condal de Lemos.

\section{BiBLIOgRAFÍA}

Álvarez Álvarez, C. 1995. "Mecenazgo nobiliario y órdenes mendicantes en la diócesis de Astorga (1250-1500)", en El Monacato en la diócesis de Astorga durante la Edad Media, Actas del Congreso: 117-144. Astorga.

Arias, M. C. 1994. "Doña María de Toledo y su obra”, en Las Clarisas en España y Portugal, Congreso Internacional, Salamanca, 20-25 de septiembre de 1993, vol. I: 343-378. Madrid.

Balboa de Paz, J. A. 2005 (2aed.). El monasterio de Carracedo. León.

Campos Sánchez-Bordona, D. 1993. "Los esgrafiados del convento de Nuestra Señora de la Concepción de Villafranca del Bierzo (León)", Estudios Humanísticos. Geografía, Historia, Arte, 15: 207-224.

Cavero Domínguez, G. 1990. "Fundaciones concepcionistas bercianas (siglo XVI)", en La orden concepcionista. Actas del I Congreso Internacional, vol. I: 411-426, León.

Cavero Domínguez, G. 1992. "El monasterio cisterciense de San Miguel de las Dueñas. La crisis del siglo XV", en Actas del Congreso Internacional sobre San Bernardo e o Císter en Galicia e Portugal, vol. II: 733-744. Ourense.

Cavero Domínguez, G. 1994. Catálogo del Monasterio de San Miguel de las Dueñas, dentro de éste se encuentra "Monasterio de San Guillermo de Villabuena (1172-1527). León.

Cavero Domínguez, G. y Domínguez Sánchez, S. 2000. Colección Documental de la Catedral de Astorga (1300-1499), vol. III, Astorga.

Cavero Domínguez, G. y González García, M. A. 2000. El monasterio cisterciense de San Miguel de las Dueñas: 197-198, León.

Cavero Domínguez, G., Álvarez Álvarez, C. y Martín Fuertes, J. A. 2001. Colección Documental del Archivo Diocesano de Astorga. León.
De Herrezuelo, Joaquín, Historia del monasterio de San Pedro de Montes, manuscrito que se conserva en Archivo Diocesano de Astorga, ms. 24-10.

De la Vega, Malaquías, Chronologia de los llustrisimos Jueces de Castilla, Nuño Peres Pesura y Layn Calvo; antecesores de la esclarecida familia de Castro; condes de Andrade, Lemos y Villava y marqueses de Sarria; de cuyos progenitores y sucesores por lineas derechas y transversales se tratan en este, Biblioteca Nacional, ms. 19.418, fol. 369r.

De Llamas, Jerónimo. 1993. Fundación y dotación del monasterio de Carracedo, año 1593, ed. de F. González González, Zaragoza.

Delgado, Ambrosio, Historia de San Miguel de las Dueñas, manuscrito conservado en el Archivo del monasterio del mismo nombre.

Fernández Vázquez, V. 2007. El señorío y marquesado de Villafranca del Bierzo a través de la documentación del Archivo Ducal de Medina Sidonia, Ponferrada.

Franco Silva, A. 1981 "Bienes, rentas y vasallos del Señorío de Villafranca del Bierzo", Archivos Leoneses, 69: 39-72.

Franco Silva, A. 1982 "El Señorío de Villafranca del Bierzo", Boletín de la Real Academia de la Historia, CLXXIX: 35-160.

Gancedo Sandes, D. 1995. "El convento de San Francisco de Villafranca del Bierzo. Siglo XV", en El Monacato en la diócesis de Astorga durante la Edad Media, Actas del Congreso: 269-275. Astorga.

Gancedo Sandes, D. 2001. "El espacio urbano de Villafranca", Revista del Instituto de Estudios Bercianos, 27: 5-68.

García Lobo, V. 1996. "La epigrafía en Carracedo", en Monasterio de Santa María de Carracedo: 67-68. León.

García Lobo, V. 1997. “De epigrafía cisterciense. Las inscripciones del monasterio de Carracedo", Cistercium 208: 189-205.

García Tato, I., 2008. Doña Beatriz de Castro, Ponferrada.

Gómez Bajo, M. C. 1994. Fondo documental del priorato de Santa María de Cluny de Villafranca del Bierzo, siglos XIV-XV, Ponferrada.

González González, F. (ed.) 1993. Memorial de la fundación y dotación del antiguo y devoto monasterio de Sancta María de Carracedo, Zaragoza.

Jiménez Suárez, M. J. 2005. Colección documental de San Andrés de Espinareda (1043-1428), León.

Martín Fuertes, J. A. 1979. "Don Luis Osorio, caballero y prelado leonés del siglo xv. Esbozo biográfico", Estudios humanísticos 1: 109-120.

Martín Fuertes, J. A. 1988. De la nobleza leonesa. Los Osorio y el Marquesado de Astorga, León.

Martínez Martínez, M. 1997-1999. Cartulario de Santa María de Carracedo (992-1500) (=l C.), 2 vols., Ponferrada: Instituto de Estudios Bercianos.

Pardo de Guevara y Valdés, E. 2000. Los señores de Galicia: tenentes y condes de Lemos en la Edad Media, Santiago de Compostela: Fundación Pedró Barrié de la Maza.

Pardo de Guevara y Valdés, E. 2012. Los linajes, parentelas y grupos de poder. Aportaciones a la historia social de la nobleza bajomedieval gallega, Madrid.

Quintana Prieto, A. 1971. Tumbo viejo de San Pedro de Montes, León.

Rodríguez González, M. C. 1992. Economía y poder en El Bierzo del siglo XV. San Andrés de Espinareda. Universidad de SantiagoUniversidad de León.

Santos Díez, J., 1961. La Encomienda de monasterios en la Corona de Castilla, siglos $X-X V$, Roma.

Velasco, Genadio, Memoria de las advertencias que conviene saber al que governare esta casa de San Pedro de Montes, manuscrito que se conserva en el Archivo Diocesano de Astorga, ms. 24-11.

VV. AA. 2006. San Pedro de Montes. Luz en la memoria. Actas. Ponferrada: Instituto de Estudios Bercianos.

Yepes, A. 1617. Coronica de la orden de San Benito, vol. III. Valladolid.

89 Ha sido estudiada por Campos Sánchez-Bordona, D. 1993. "Los esgrafiados del convento de Nuestra Señora de la Concepción de Villafranca del Bierzo (León)", Estudios Humanísticos. Geografía, Historia, Arte, 15: 207-224. 